\title{
Biochemical, Biophysical and Mechanical Characterization of Decellularized Dermal Implants
}

\author{
Frederick H. Silver ${ }^{1}$, Dale DeVore ${ }^{2}$, Ruchit Shah ${ }^{3}$ \\ ${ }^{1}$ Department of Pathology and Laboratory Medicine, Robert Wood Johnson Medical School, Rutgers, the State University of New \\ Jersey, Piscataway, NJ, USA \\ ${ }^{2}$ DV Consulting Services, Chelmsford, MA, USA \\ ${ }^{3}$ Graduate Program in Biomedical Engineering, Rutgers, the State University of New Jersey, Piscataway, NJ, USA \\ Email: fhsilver@hotmail.com
}

How to cite this paper: Silver, F.H., DeVore, D. and Shah, R. (2017) Biochemical, Biophysical and Mechanical Characterization of Decellularized Dermal Implants. Materials Sciences and Applications, 8, 873-888.

https://doi.org/10.4236/msa.2017.812064

Received: August 8, 2017

Accepted: November 7, 2017

Published: November 10, 2017

Copyright (c) 2017 by authors and Scientific Research Publishing Inc. This work is licensed under the Creative Commons Attribution International License (CC BY 4.0).

http://creativecommons.org/licenses/by/4.0/

\begin{abstract}
Allografts have been used in a variety of applications to augment as well as replace tissues throughout the body. A number of steps are involved in selection, harvesting, processing and testing of dermal allografts. Grafts can be obtained that are: free of antibodies to viruses and low in viral titers. Cellular material can be eliminated from the tissue and the product becomes almost exclusively a collagen fiber network. The purpose of this paper is to examine the changes in collagen structure and properties that occur during processing of decellularized dermis. The results indicate that collagen fiber swelling occurs during processing although the product preserves the native collagen banding pattern at the fibrillar structural level. Fiber swelling and decreased collagen deformability of processed dermis, may lead to stress concentration at the implant-tissue interface and up-regulation of mechanotransduction. This may lead to premature mechanical failure due to creation of a chronic inflammatory condition at the implant-tissue interface. It is suggested that all dermal allografts be oriented such that Langer's lines of the implant match those of the host tissue, and that wound closure by suturing be done under conditions that preserve the normal tension in skin in order to minimize implant-interfacial failure.
\end{abstract}

\section{Keywords}

Collagen, Dermis, Repair, Wound Healing, Optical Coherence Tomography, Vibrational Analysis, Resonant Frequency, Modulus, Langer's Lines, Mechanotransduction 


\section{Introduction}

Synthetic materials have gained widespread use as replacements for damaged tissues in a wide variety of applications [1] [2]. Allografts and xenografts have grown appreciably in their use in applications that include: bladder regeneration [3], burns [4], breast reconstruction [5], facial cosmetic defects [6], free flap coverage [7], hernias [8], ophthalmic defects [9], oral defects [10], replacement of cardiovascular tissues [11], tendon/ligament repair [12], and vaginal repair [13]. They also have been used as replacements in the repair of rotator cuff, Achilles and quadriceps tendons, primary staged and revised breast reconstruction, hernia repair, treatment of chronic wounds such as diabetic foot ulcers and pulmonary patches [14] [15] [16].

While synthetic materials such as Dacron and Teflon work well in many applications, and stainless steel and other metal wires are useful in stents, natural materials such as auto- and allografts are useful in many applications where cellular ingrowth and repair responses are required to promote long-term healing of tissues such as dermis and ligaments [17] [18]. While many studies underscore the utility of dermal allografts, questions arise regarding the effects of processing conditions such as decellularization, decontamination, and viral deactivation on the structure of these tissues and the surgical outcome. In addition, more than one third of hernia replacements require revision surgery. Failures of these implants typically occur at the implant-tissue interface due to mechanical mismatches [16]. Therefore, the mechanical properties of allograft materials appear to be an important characteristic needed for a positive outcome at least in hernia repair.

The primary component of allograft tissue is extracellular matrix (ECM) composed of collagen and elastic fibers, proteoglycans, cell attachment factors, growth factors, signaling molecules, cells, ions and water [2]. The 3-D structure of collagen scaffolds has been shown to promote the repair response in dermal and orthopedic applications by minimizing the deposition of granulation tissue and promoting organized collagen deposition along the major axis of the scaffold [17] [18].

Use of tissue derived collagen scaffolds for human surgical applications requires removal of cellular components of the epidermis and dermis and processing into sheets or other forms. During removal of cells from tissues, changes in the physical structure and mechanical properties occur that may affect the resulting strength and healing properties of these materials. Moore et al. reported the effects of using a non-denaturing anionic detergent, recombinant endonuclease and antibiotics on the decellularization and mechanical properties of human dermis [14]. They showed that $97 \%$ of the DNA was removed as well as cell membrane components such as major histocompatibility complex-I yielding materials with ultimate loads of up to $635 \mathrm{~N}$. Terzini et al. reported decellularization in $0.06 \mathrm{~N} \mathrm{NaOH}$ for 1 to 7 weeks at room temperature [19]. They found that the ultimate stress of the product was between 4 and $10 \mathrm{MPa}$ between 
5 and 6 weeks of treatment. Longer treatments reduced the strength. It is important to understand how processing conditions affect the physical structure and properties of decellularized dermal implants.

The purpose of this paper is to review typical steps involved in selection and processing of human dermal tissues for use in surgery. These steps include donor selection, removal of the epidermis and dermal cells, viral deactivation, and tissue processing. The influence of processing on the structure and mechanical properties of dermal collagen scaffolds is examined in this paper.

\section{Methods}

\subsection{Patient Selection}

Human skin was obtained from the tissue banks that comply with all American Association of Tissue Banks (AATB) standards and FDA regulations CRF 21 Parts 1270 and 1271. All donors were qualified based on serological testing, medical/social history, neurological screening and physical assessment. All donors were de-identified by the tissue bank and only sample numbers were provided for identification. Serology testing was conducted for antibodies to HIV-1, HIV-2, Hepatitis A and B, HTLV-1 (human T-lymphotropic Virus), pseudorabies virus (PRV), porcine parvovirus (PPV), bovine diarrhea virus (BVD) and protein-resistant prion disease (PRPD). Samples with antibodies to any of these markers were discarded. Samples were rejected from patients with any of the following neurologically related diseases or conditions: Creutzfeldt-Jacob disease, pituitary disease, dura mater implantation, degenerative or demyelinating disease or death in a neurological or psychiatric hospital. Physical assessment of donor skin included evaluation of the risk of a sexually transmitted disease, presence of needle tracks or tattoos, unexplained jaundice and signs of infection. Any of these conditions resulted in rejection of the donated skin.

\subsection{Skin Processing}

Decellularized dermis was prepared from human skin by removal of the epidermis and dermal cells, viral inactivation, freezing, and freeze drying. The epidermis was removed by immersion in $2 \mathrm{M} \mathrm{NaCl}$ for 24 to 27 hours at temperatures between $2^{\circ} \mathrm{C}$ and $8^{\circ} \mathrm{C}$. The epidermis was then scraped with a sterilized spatula until all the loose material was removed. Viral inactivation was achieved by immersion of the dermis in $0.5 \mathrm{M} \mathrm{NaOH}$ at $\mathrm{pH}=12.0$ (high $\mathrm{pH}$ ) for 60 minutes at temperatures between $4^{\circ} \mathrm{C}$ and $10^{\circ} \mathrm{C}$ by adding $6 \mathrm{ml} \mathrm{NaOH}$ solution per gram of tissue. The tissue is then washed in $0.05 \mathrm{M}$ phosphate buffer $\mathrm{pH}=6.5$ three times and then the $\mathrm{pH}$ is lowered to 2.5 (low $\mathrm{pH}$ ) using $0.5 \mathrm{M}$ phosphoric acid for three hours at between $2^{\circ} \mathrm{C}$ and $8^{\circ} \mathrm{C}$. The $\mathrm{pH}$ of the dermis is then raised to 6.5 by adding $0.5 \mathrm{M} \mathrm{NaOH}$. One gram of dermis is then placed in $6 \mathrm{ml}$ of reagent alcohol (90\% ethanol, 5\% isopropanol, 5\% methanol) and the material is placed at $2^{\circ} \mathrm{C}$ to $8^{\circ} \mathrm{C}$ for 1 to 14 days until the viral count is reduced to an acceptable level. The material is then washed with distilled water, frozen, and freeze dried. 


\subsection{Biochemical Analysis}

Four by four $\mathrm{mm}$ pieces of decellularized dermis are placed in $0.02 \mathrm{M}$ dibasic sodium phosphate buffer at $\mathrm{pH} 9.5$ and are fragmented with an in-line rotor/ stator macerator. The jacket of the incubator is cooled to $1^{\circ} \mathrm{C}$ by pumping cold water through it. Glutaric anhydride at $50 \mathrm{mg} / \mathrm{ml}$ in isopropyl alcohol is added to the mixture to a final concentration of $0.2 \% \mathrm{~W} / \mathrm{V}$ to solubilize the tissue. The final $\mathrm{pH}$ is adjusted to 9.5 using $0.5 \mathrm{M} \mathrm{NaOH}$. SDS PAGE gel electrophoresis was conducted on dermal samples to determine the collagen types present using the methods reported previously [20].

\subsection{Light Microscopy, Morphological Analysis and Transmission Electron Microscopy}

Gross morphology was obtained by taking photographs of harvested skin at magnifications between 4 and 40x. Morphological observations are made on samples that were cut from unfrozen dermis and decellularized tissue that were stored in a 60/40 (W/V) alcohol/water mixture and then sent to Pacific Pathology (San Diego, CA) for processing. Longitudinal and cross sections of the samples were stained with Hematoxylin and Eosin (H \& E) and were observed under a light microscope. Samples were evaluated for normal collagen architecture. Low magnification pictures were taken at $4 \times$ and then the image was scanned and serial sections were reconstructed in $3 \mathrm{D}$ to produce an image of the whole tissue to determine the fiber diameters and lengths. The collagen fiber diameter and length have been shown to be related to the strength of collagenous tissues [21].

\subsection{Transmission Electron Microscopy}

Collagen fibrils were solubilized from processed dermis as described above for biochemical analysis, placed on carbon-coated copper grids and stained with saturated aqueous uranyl acetate for $5 \mathrm{~min}$. The $\mathrm{pH}$ of the unbuffered staining solution was 3.8. Stained fibrils were viewed with a Philips 300 transmission electron microscopy at a magnification of $60,000 \times$ as described previously [22].

\subsection{Image Analysis}

Images of $\mathrm{H} \& \mathrm{E}$-stained histological sections of dermis were are acquired at 4 and $40 \times$ magnifications, with four images captured for each section. The images were then imported into the graphics editing program, Adobe Photoshop, and the color and brightness of each were adjusted for uniformity. The images were then rotated, cropped and knit together, and then the compiled image is adjusted for higher contrast between the two dye colors resulting from $\mathrm{H} \& \mathrm{E}$ staining.

Portions of whole sections were used to create models and extrapolate data, using Adobe Photoshop, Adobe Illustrator, and ImageJ, a NIH-developed image processing program, as well as Solidworks, a 3-dimensional computer-aided de- 
sign program.

Smaller, representative sections of the images were established and imported into Adobe Illustrator, a vector graphics design program. This program was used to convert the current images, in rasterized, or pixilated form, to vector images, and enables computer-aided design programs to recognize each individual shape as an entity. The vectorized images were then imported into Solidworks, a computer-aided design (CAD) program. The vector files were then converted into CAD model files, with the chosen images being approximately $1 \mathrm{~mm}$ in length and width, and $6 \mu \mathrm{m}$ in thickness. Because of the limitations of Solidworks, the models were scaled up 225 times in all dimensions.

\subsection{Enzyme Degradation Studies}

Enzyme degradation was measured by immersing $0.1 \mathrm{~g}$ of wet dermis into buffered solutions containing one of the following enzymes: 1) bacterial collagenase, or 2) pepsin. Collagen degradation due to exposure to denaturing conditions will decrease the resistance to bacterial collagenase. If degradation is severe then the resistance to pepsin, which normally only degrades the non-helical ends of the collagen is lowered. Samples are incubated for 4.8 hours with the enzymes and then the amount of sample remaining is determined gravimetrically. Normally a $0.1 \mathrm{~g}$ weight of wet tendon contains about $25 \%$ to $30 \%$ tissue when dried. This means that that a $0.1 \mathrm{~g}$ sample of dermis has about 0.025 to $0.03 \mathrm{~g}$ of tissue before digestion.

The fraction of collagenous tissue that remains after digestion was determined using $1000 \mathrm{U} / \mathrm{ml}$ of bacterial collagenase (Sigma-Aldrich) in PBS pH $=7.4$ containing $25 \mathrm{mM} \mathrm{CaCl}_{2}$. Tissue samples were incubated at $37^{\circ} \mathrm{C} \pm 2^{\circ} \mathrm{C}$ for 4.8 hours, removed from incubator and $1 \mathrm{~mL}$ of $10 \mathrm{mM}$ EDTA solution was added to stop the enzyme reaction. The digested material was separated from undigested material, washed with DI water, air dried for $24 \mathrm{hrs}$ and then weighed. The fraction of undigested tissue was calculated by dividing the final weight by 0.1. Material removed during collagenase digestion consists of collagen damaged during processing.

Pepsin digestion was accomplished by mixing $0.1 \mathrm{~g}$ of tissue samples with $5 \mathrm{ml}$ pepsin (Sigma-Aldrich) solution $(6 \mathrm{mg} / \mathrm{ml}$ in $\mathrm{HCl} \mathrm{pH}=2.5)$, and incubating the mixture for $4 \mathrm{hrs}$ at $22^{\circ} \mathrm{C}$. At that time the undigested material was washed with $5 \mathrm{ml}$ of DI water two times and then the samples were air dried for $24 \mathrm{hrs}$ at $37^{\circ} \mathrm{C}$. The dried sample was weighed and the number was divided by 0.1 to get the fraction of tissue remaining after pepsin digestion. The fraction of pepsin insoluble material sometimes increases after processing since processing may remove pepsin soluble tissue fragments.

\subsection{Mechanical Testing}

Decellularized human dermal samples were tested after immersion in phosphate buffer solution as described elsewhere [23] [24] [25]. Both tensile stress-strain 
test and vibrational optical cohesion tomography (OCT) were used to measure the modulus of all of the samples. Samples were tested wet after soaking in phosphate buffer solution at $\mathrm{pH} 7.4$ for at least 30 minutes. Processing and testing steps were conducted at $22^{\circ} \mathrm{C}$.

\subsection{OCT and Vibrational Analysis in vitro}

Determination of the modulus using OCT and vibrational analysis was conducted as discussed previously [23] [24] [25]. The resonant frequency, $f_{n}$, of each sample was measured and converted into a modulus value using Equation (1),

$$
E=m L\left(2 \pi f_{n}\right)^{2} / A
$$

where $m, L$ and $A$ are the sample mass, length and cross-sectional area.

The relationship between the modulus measured using vibrational and tensile measurements is given by Equation (2)

$$
E v=1.026 E t+0.0046
$$

where, $E_{V}$ and $E t$ are the moduli measured using vibrational and tensile measurements, respectively and are in MPas [23] [24] [25].

\section{Results}

\subsection{Serological Testing and Biochemical Analysis}

Serological testing was conducted to establish the removal of viruses that may contaminate dermal allografts. Table 1 shows the results of viral testing for HIV, Hep A, PPV, PRV and BVDV. Results of serological testing were negative for antibodies to HIV-1, HIV-2, Hepatitis B and HTLV-Y. Viral inactivation studies indicated that viral inactivation lowered the log of the viral titers from about 3.77 to as much as 10.3 .

SDS PAGE electrophoresis results on solubilized dermal tissue showed that it contained Types I, III and VI collagens as well as fibronectin.

\subsection{Light Microscopy, Histology and Electron Microscopy}

Light microscopic studies illustrated that the freshly harvested skin was composed of a shiny epidermis on top of a pinkish dermis (Figure 1). The harvested skin pieces were about $9 \mathrm{~cm}$ wide and $12 \mathrm{~cm}$ long.

Table 1. Results of serological testing on skin allografts before decellularization.

\begin{tabular}{cccccc}
\hline \multirow{2}{*}{$\begin{array}{c}\text { Inactive } \\
\text { solution }\end{array}$} & HIV & Hepatitis A & PPV & PRV & BVDV \\
\cline { 2 - 5 } $\mathrm{pH}>12$ & $>4.89$ & 3.97 & $>5.06$ & $>4.09$ & $>3.77$ \\
$\mathrm{pH}<1.0$ & & $>2.2$ & 5.24 & $>5.5$ & \\
$\mathrm{pH}<2.5$ & 4.0 & & & 6.24 & $>3.77$ \\
Total log reduction & $>8.89$ & $>6.17$ & $>10.3$ & $>9.59$ & \\
\hline
\end{tabular}




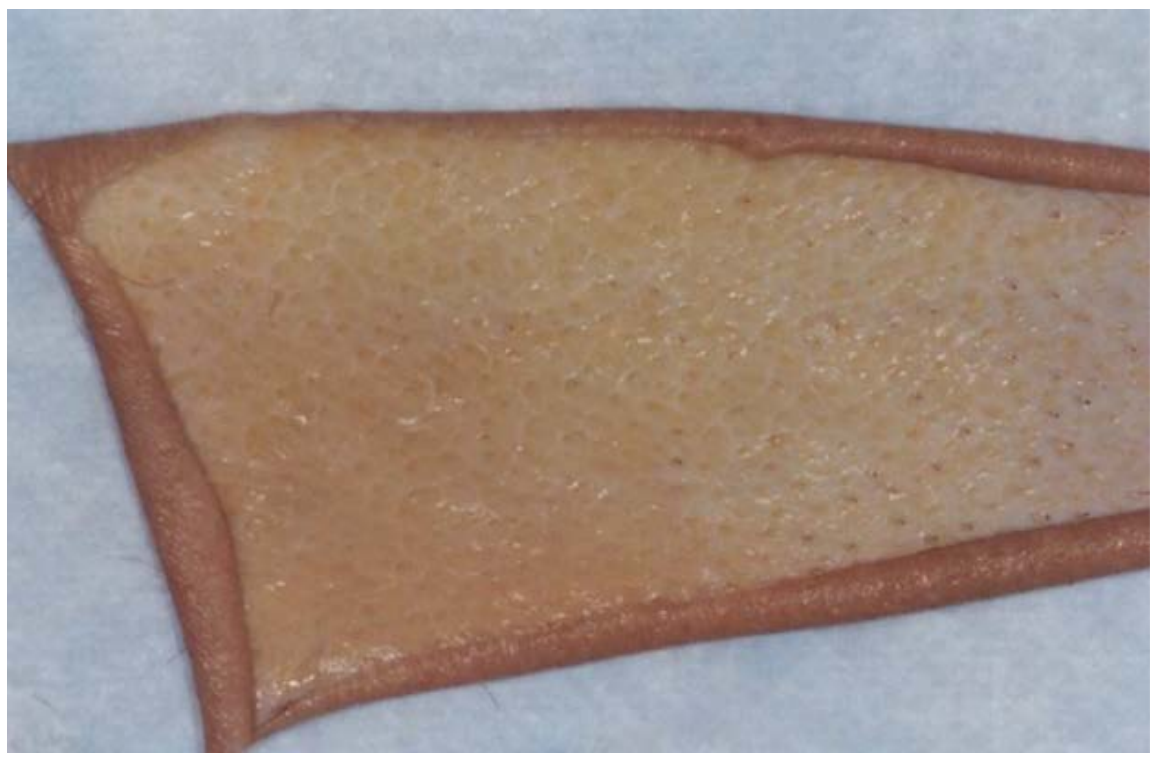

Figure 1. Photographic image of freshly harvested human skin. Harvested skin has a shiny appearance at the epidermal surface while dermis appears pink before processing.

Results of histologic studies indicated that the harvested skin had a thin epidermis on top of the dermis that was separated from the dermis after treatment with the solution containing $2 \mathrm{M} \mathrm{NaCl}$ (Figure 2). After viral inactivation and decellularization, very few cells were visible by light microscopy; however, the collagen matrix appeared to stain less intensely and appeared swollen when compared to the control dermis (Figure 3). Transmission electron microscopy indicated that collagen fibrils had the normal light-dark repeat pattern characteristic of type I collagen fibrils with no evidence of cells or proteoglycans present between the collagen fibrils (Figure 4).

\subsection{Image Analysis and Enzyme Digestion}

Image analysis was conducted on serial sections reconstructed into a montage of the whole sample that was a composite of several stained histological sections (Figure 5). When unprocessed skin was compared to processed dermis, it was clear that the collagen fibers of the processed dermis were swollen (see Figure 6). When the cross-sectional area of the unprocessed dermal collagen fibers were quantitatively compared to those of processed dermal fibers, the latter was much larger based on image analysis results (see Table 2).

When unprocessed and processed dermal samples were treated with bacterial collagenase and pepsin, the $\%$ of the sample remaining after collagenase digestion increased while the $\%$ of the sample remaining after pepsin digestion decreased after processing (Table 3).

\subsection{Mechanical Testing}

Moduli values for decellularized dermis were obtained based on a combination of optical coherence tomography and vibrational analysis as previously 


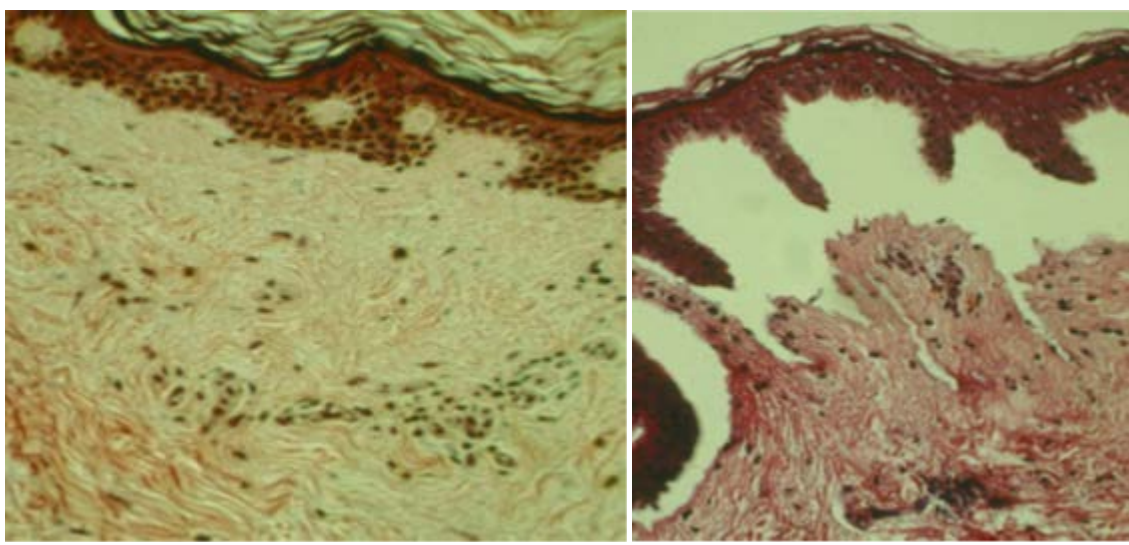

Figure 2. Histological images of human skin before and after separation of epidermis from the dermis. Histological images of $\mathrm{H} \& \mathrm{E}$ stained sections of unprocessed skin showing epidermis on the top surface (left) and skin treated with $2 \mathrm{M} \mathrm{NaCl}$ (right) to remove the epidermis. Note the loose epidermis is scraped away from the dermis before the dermis is further processed.
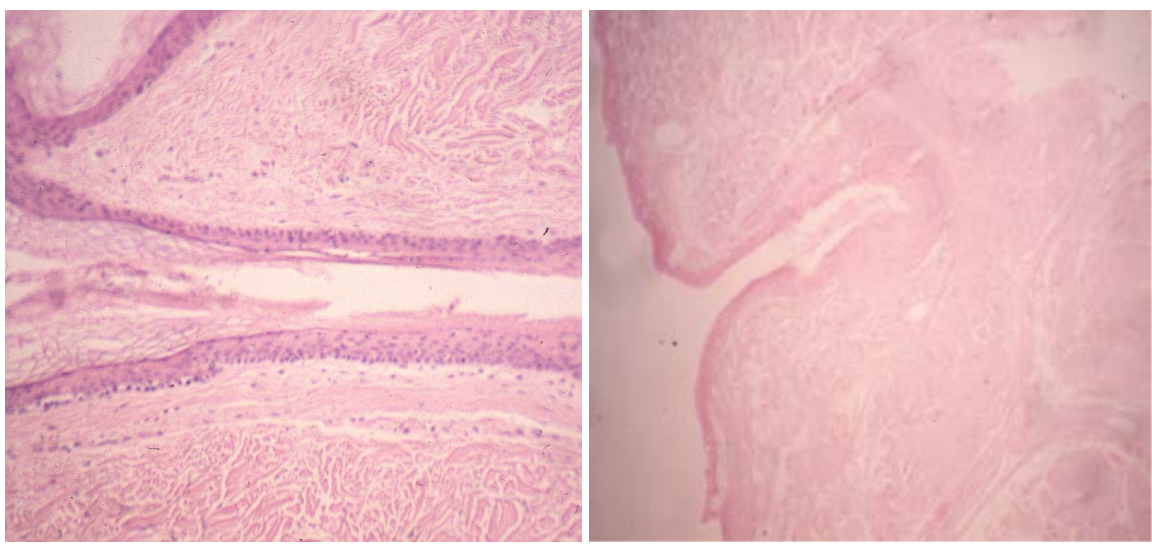

Figure 3. Histological evaluation of human dermis before and after removal of dermal cells. Intact dermis (left panel) stained with $\mathrm{H} \& \mathrm{E}$ (hematoxylin and eosin) shows the presence of many dark nuclei while the treated dermis shows that the section is devoid of dark nuclei after treatment to remove cellular material (right panel). Notice the relatively weak staining intensity of the collagen in the decellularized dermis indicating that the collagen fibers are less dense after removal of the cells.

Table 2. The summary of quantitative data acquired from the images in Figure 6 obtained from image analysis.

\begin{tabular}{ccccc}
\hline Skin state & $\begin{array}{c}\text { Number of } \\
\text { fibers }\end{array}$ & Average area $(\mu \mathrm{m})$ & \multicolumn{2}{c}{ Average fiber length $(\mu \mathrm{m})$} \\
\hline Unprocessed & 966 & 0.000162 & 0.0294 & y-direction \\
Processed & 518 & 36.125483 & 0.0310 & 0.0156 \\
\hline
\end{tabular}

described [23] [24] [25]. A calibration curve of modulus determined from vibrational measurements and that obtained from standard tensile measurements is shown in Figure 7. The modulus from vibrational measurements was calculated by measuring the resonant frequency of decellularized dermis (Figure 8) and use 


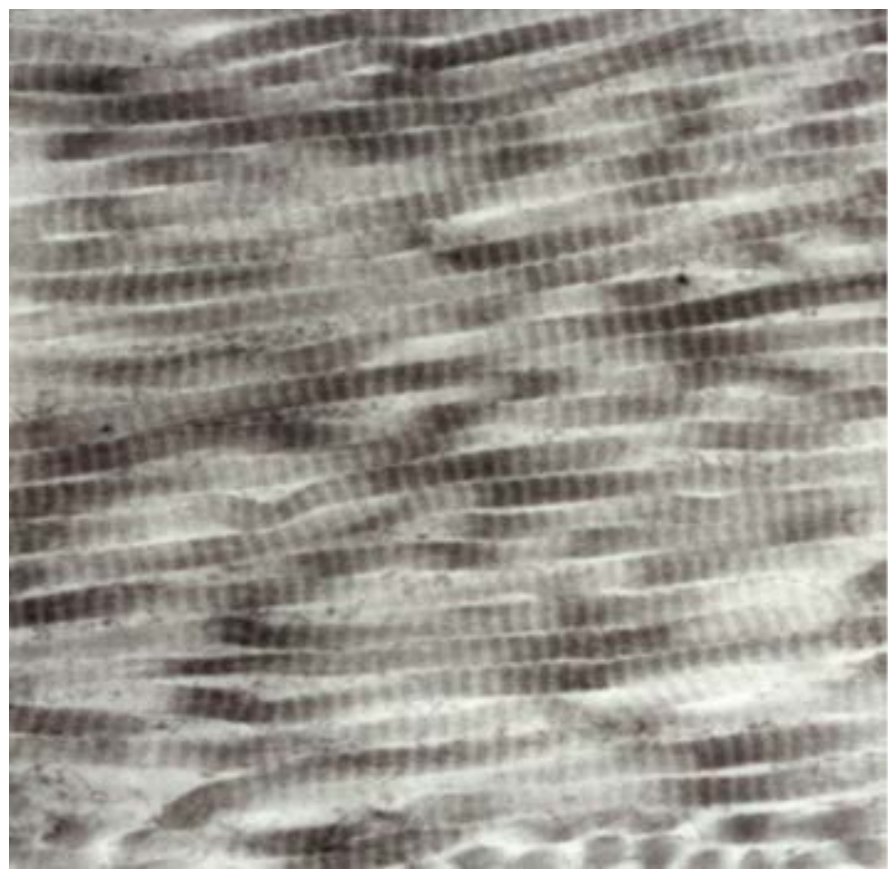

Figure 4. Transmission electron micrograph of dermal collagen fibrils from decellularized human tissue. The image shows negatively stained collagen fibrils found in the dermis of decellularized tissue after treatment to remove the cellular material. Note the sample is composed of collagen fibrils with no evidence of cellular debris or proteoglycans present.
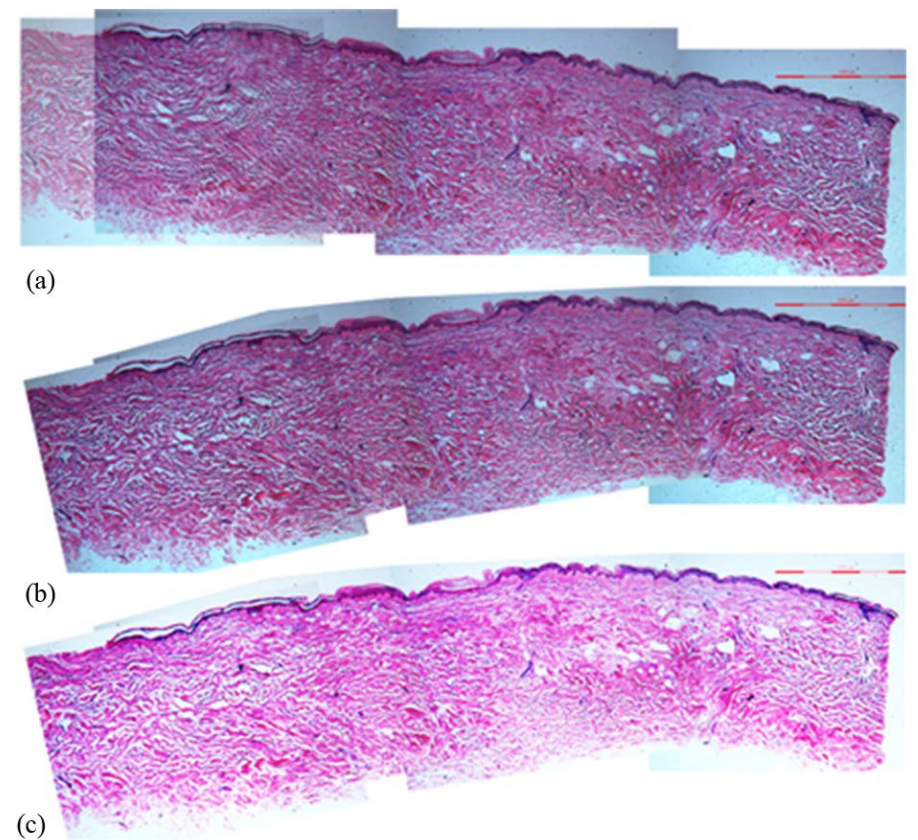

Figure 5. Montage showing the images of serial sections of unprocessed human skin. The images of the original unprocessed section of skin: (a) all four images rotated and cut to fit together; (b) brightness/color/contrast balanced; and (c) contrast/color adjusted to accentuate the differences in the $\mathrm{H} \& \mathrm{E}$ stains. Note the darker epidermis is present at the surface and appears to be sloughing off. The dermis is characterized by a multitude of pinkish collagen fibers. 


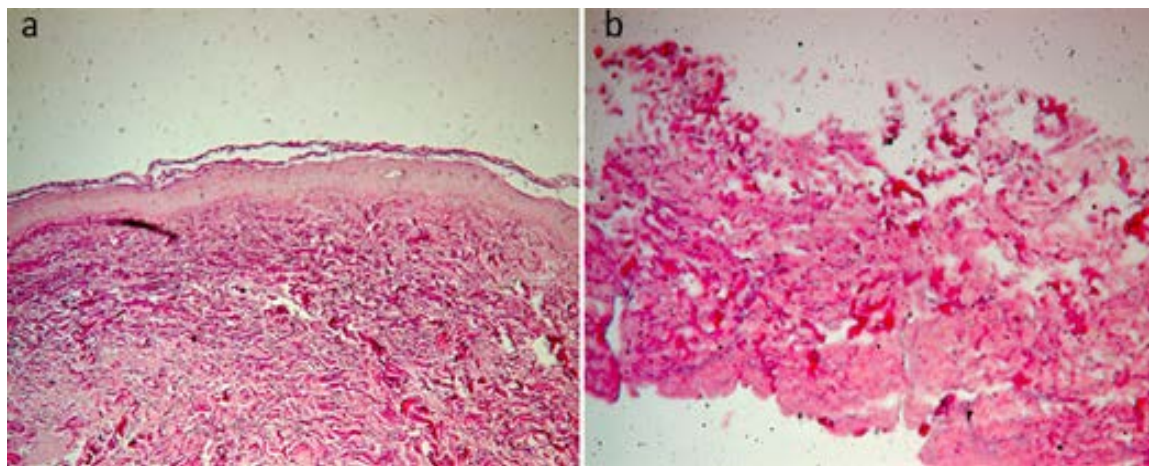

Figure 6. H \& E stained sections of skin and processed dermis. The images of sections of: (a) unprocessed skin showing the presence of the darker staining epidermis at the top and (b) processed dermis showing that the collagen fibers (pink) appear to be swollen and less dense.

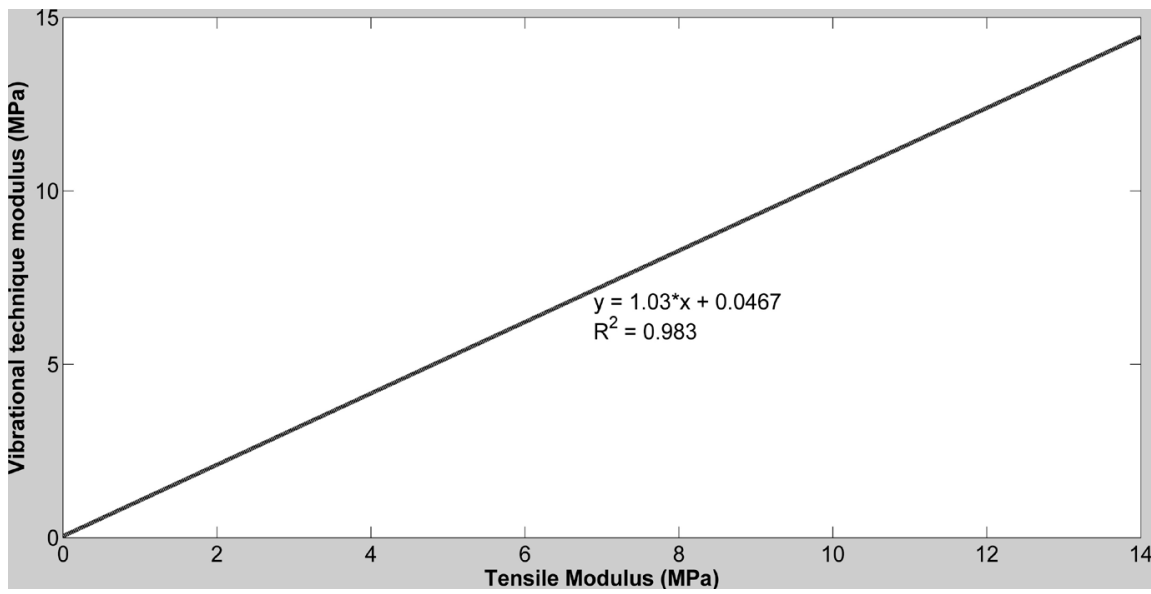

Figure 7. Calibration curve of modulus determined from vibrational studies and conventional tensile testing. This figure was modified based on Shah et al. [23] [24] [25]

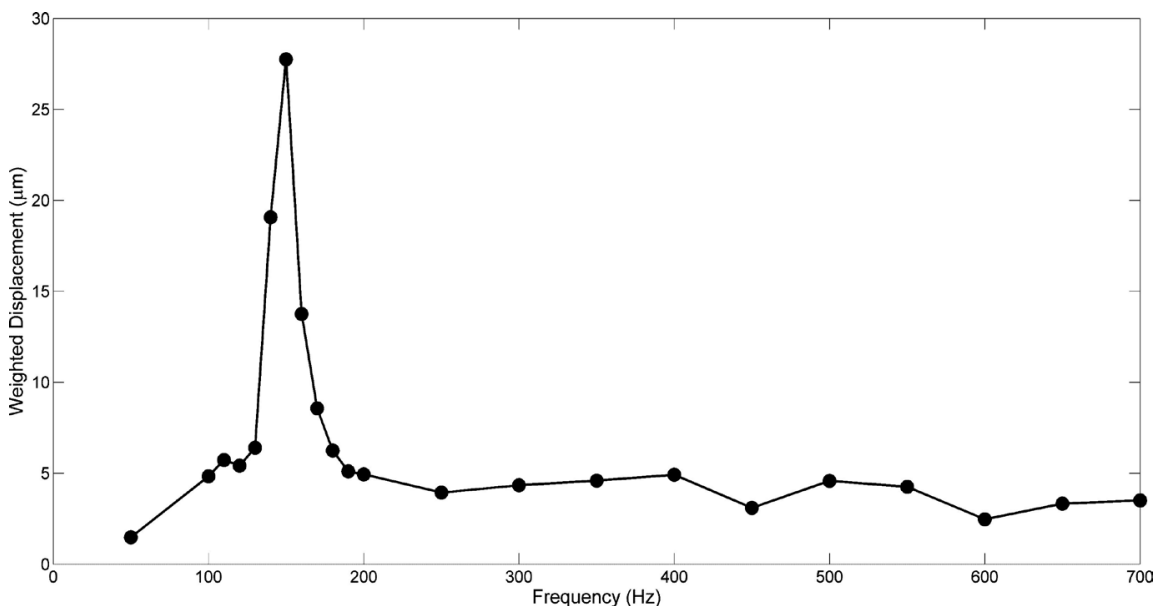

Figure 8. Vibrational analysis of decellularized dermis. Determination of the resonant frequency from measurement of the maximum displacement of a sample vibrated between 0 and $700 \mathrm{HZ}$. Note the maximum displacement is measured and converted into a vibrational modulus using Equation (1). The modulus calculated from vibrational studies was calculated from the resonant frequency as described by Shah et al. [23] [24] [25]. 
of Equation (1). The maximum modulus measured for decellularized dermis was about $17 \mathrm{MPa}$ as was previously reported [24] [25] [26]. Modulus measurements on made on decellularized dermis are strongly dependent on strain (Figure 9) as previously reported [24] [25]. However, they also depend on the direction of Langer's lines (Table 4).

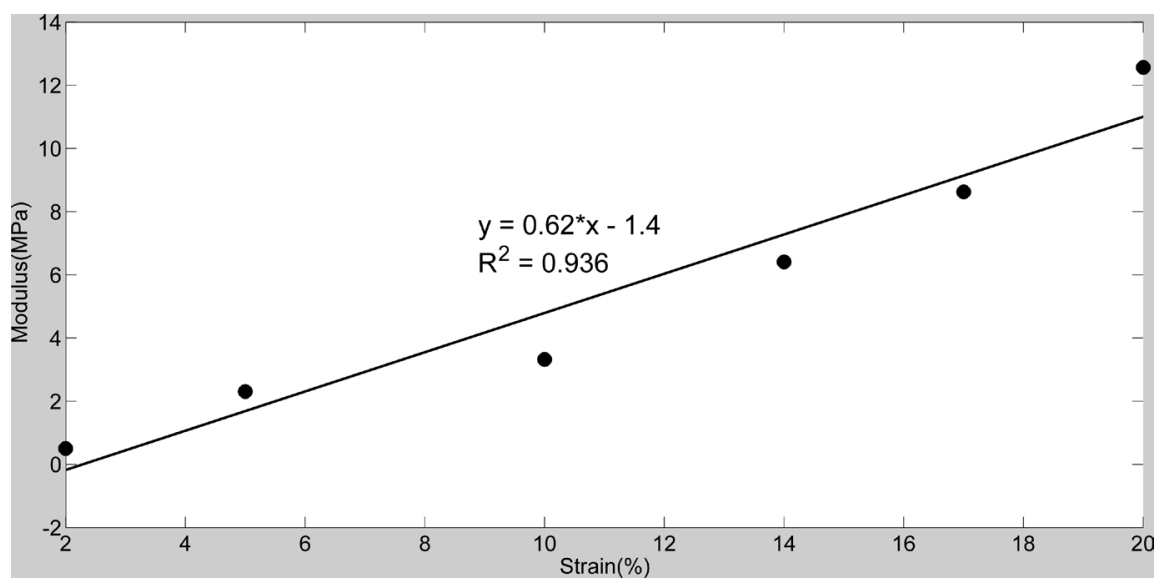

Figure 9. Plot of modulus determined from OCT and vibrational studies versus strain for decellularized dermis. Note the resonant frequency and modulus increase with strain as previously reported [23] [24] [25].

Table 3. Collagenase and pepsin digestion results for unprocessed (UN) and processed (P) human dermis.

\begin{tabular}{cccc}
\hline Sample & Status & $*$ *ollagenase \% (SD) & $*$ Pepsin \% (SD) \\
\hline $\mathbf{1}$ & UN & $16.98(0.55)$ & $25.0(0.60)$ \\
& P & $13.98(0.67)$ & $43.1(1.90)$ \\
2 & UN & $24.60(2.87)$ & $33.4(0.67)$ \\
& P & $10.78(2.04)$ & $28.7(0.40)$ \\
& UN & $18.20(0.59)$ & $46.0(1.60)$ \\
\hline
\end{tabular}

*\% of dry weight remaining after enzyme digestion of a $0.1 \mathrm{~g}$ wet sample-normal skin contains $25 \%$ to $30 \%$ collagen by dry weight.

Table 4. Modulus measurements made using OCT and vibrational analysis along and perpendicular to Langer's Lines (LL) in processed decellularized dermis. Note decellularized dermis fails perpendicular to LL at strains as low as $10 \%$.

\begin{tabular}{ccc}
\hline \multirow{2}{*}{ Strain (\%) } & \multicolumn{2}{c}{ Modulus (MPa) } \\
\cline { 2 - 3 } & Parallel to LL & Perpendicular to LL \\
\hline $\mathbf{2}$ & $2.09+/-0.24$ & $0.69+/-0.04$ \\
$\mathbf{5}$ & $2.54+/-0.27$ & $0.95+/-0.48$ \\
$\mathbf{8}$ & $4.80+/-0.37$ & $1.63+/-0.063$ \\
\hline
\end{tabular}


Modulus measurements made along and perpendicular to Langer's Lines indicate that decellularized dermis is stiffer along Langer's Lines as opposed to perpendicular to them (Table 4) as expected. In addition, although the strain to failure of decellularized dermis is about $20 \%$ along Langer's Lines that of intact skin is much greater $(40 \%-80 \%)$. The strain at failure in the direction perpendicular to Langer's lines ( $10 \%$ to $15 \%$ ) is less than that along Langer's lines (about $20 \%$ to $25 \%$ ) indicating that the orientation of collagen fibers in allograft tissue may be an important consideration when minimizing the strain at the host-tissue interface. This is due to the large difference in deformability of skin $(40 \%-80 \%)$ and processed dermis (10\% to $25 \%)$.

\section{Discussion}

The use of allograft tissues to replace or repair damaged tissues and organs has a long history. Allograft and xenograft dermis is used in many applications and accounts for a large volume of medical procedures. When used as filler without mechanical requirements, such as in the breast or in the face, there are few complications as long as the cellular components of the tissue are removed, the graft does not contain antibodies to viral components, the viral load, is reduced and the implant does not cause pressure on the surrounding tissue.

In applications that are structural, such as in repair of herniated abdominal wall, the mechanical properties of the graft material play an important role [16]. The results of recent studies suggest that allograft failure can be due to mechanical mismatches between graft and the host tissues [16]. It is known that intimal hyperplasia occurs with vascular grafts due to mechanical mismatch between a low modulus vessel wall and a stiff graft made of polyethylene terephthalate, Dacron [26]. Pressure induced tissue necrosis has also been reported at the femoral artery closure point in animals, and after breast reduction surgery in humans [26] [27]. This suggests that effective tissue repair using allografts should involve limiting the stress and strain at the implant-issue interface whether the graft is loaded in tension or compression.

The interfacial stress at the tissue-implant interface arises from either pressure created by the implant on the surrounding tissue or stress concentration at the interface from suturing. The stress concentration occurs when a soft material is sutured to a more rigid one. In this case when a suture is pulled taut, the softer of the materials (skin) deforms more than the rigid material (graft). If the former is the host tissue (skin) then this sets into motion mechanotransduction leading to cellular proliferation and deposition of repair tissue [26]. Complicating stress-concentration at the interface is the fact that the modulus of dermis and skin is strain dependent. Therefore as the host skin allograft is sutured in place, the strain at the interface is increased which increases the stiffness of both host and implant materials. Minimization of cellular hyperplasia and mechanotransduction in the host tissue due to strain hardening requires minimization of the interfacial stress.

Another consideration is that most allografts do not come with the direction 
of Langer's Lines marked. The significance of this is that the surgeon does not know how to line up the collagen fiber orientation in the allograft with that of the host tissue (Langer's lines). To minimize stress concentration at the interface, Langer's Lines of the allograft need to be aligned with those of the host tissue. If this does not occur then cellular invasion and remodeling of the allograft may lead to premature graft failure due stress concentrations and up-regulation of mechanotransduction [26]. In addition, since the deformability of decellularized dermis in the direction perpendicular to Langer's lines is much lower than that of normal skin ( $10 \%-15 \%$ versus in excess of $40 \%$ in skin) this will cause stress concentration and excess host tissue deformation at the implant-tissue interface.

Results presented in this paper suggest that while allograft dermis can be harvested, decellularized, treated to decrease the viral load, and processed into a form for use in surgery, there are a number of changes that occur to the dermis that occur during processing. While decellularized dermis is composed of primarily collagen with little elastic tissue, processing to remove cells swells the collagen fibers and changes their susceptibility to enzymatic digestion by bacterial collagenase. Excessive collagen fiber swelling as a result of backbone cleavage leads to decreased resistance to the enzyme collagenase. Harsh treatments such as exposure to sodium hydroxide swell the tissue, and unravel the collagen fibers; they also lead to damage to the collagen molecular backbone that decreases the graft strength and changes the handling characteristics of the tissue. The increased resistance to pepsin after processing of the tissue suggests that some of the non-collagenous proteins present in the allograft dermis are removed during processing.

We have reported results of mechanical studies on intact skin and decellularized dermis for over 30 years [23] [24] [25] [28] [29] [30]. Results of published studies suggest that the high strain modulus of skin and processed dermis are very similar and range from 16.0 to $18.4 \mathrm{MPa}$ [23] [24] [25]. Thus processing does not appear to change the high strain modulus. However, processing does appear to reduce the ability of dermis to deform at low strains. Human skin tested in tension in vitro has a very low modulus ( $0.75 \mathrm{MPa}$ or less) up to a strain of $0.4(40 \%)$ due to the elastic fibers [30]. This is compared to decellularized dermis with a modulus of $2.57 \mathrm{MPa}$ at a strain of 0.05 (5\%) [23] [24] [25]. Human skin from the hand in vivo has an estimated modulus of about $0.66 \mathrm{MPa}$ under normal skin tension [31]. Based on these data it appears that while the high strain modulus of decellularized dermis is similar to that of normal skin, decellularized dermis appears to be less deformable than normal skin. This suggests that allografts of dermis may function better as graft materials when Langer's Lines of the graft and host are parallel and care is taken to minimize the strain at the implant-host interface. The increased stiffness of decellularized dermis over normal skin at low strains may be a result of loss of the elastic fiber network during decellularization as well as swelling of the collagen fibers that make it difficult for the material to deform under tension. 


\section{Conclusions}

Much progress has been made in selection, harvesting, processing and testing of dermal allografts. Grafts can be obtained that are: free of antibodies to viruses and have low in viral titers. Cellular material can be eliminated from the tissue and the product becomes almost exclusively a collagen fiber network. However, each of these processes can change the structure and properties of the dermis.

Morphological results suggest that processing of decellularized dermis results in collagen fiber swelling while preserving the collagen native banding structure at the fibril level. Fiber swelling and decreased collagen deformability, in conjunction with the need to orient the sample with respect to Langer's Lines, may lead to stress concentration at the implant-tissue interface. Stress concentrations may lead to premature mechanical failure due to up-regulation of mechanotransduction and creation of a chronic inflammatory condition at the graft-host interface.

It is suggested that all dermal allografts be oriented with the Langer's lines of the implant parallel to those of the host tissue and that wound closure by suturing be done under conditions that preserve the normal tension in skin and limit the stress concentration at the graft-host interface.

\section{Acknowledgements}

The authors would like to thank Raya Lim, a MS degree recipient at Robert Wood Johnson Medical School, for her help with the image processing.

\section{References}

[1] Glasgold, A.I. and Silver, F.H. (1991) Applications of Biomaterials in Facial Plastic Surgery. CRC Press, Boca Raton, Fl.

[2] Silver, F.H. and Christiansen, D.L. (1999) Biomaterials Science and Biocompatibility. Springer-Verlag, N.Y.

[3] Akbal, C., Lee, S.D., Packer, S.C., Davis, M.M., Rink, R.C. and Kaefer, M. (2006) Bladder Augmentation with Acellular Dermal Biomatrix in a Diseased Animal Model. Journal of Urology, 176, 1706-1711. https://doi.org/10.1016/j.juro.2006.04.085

[4] Griffin, J.E. and Johnson, D.L. (2005) Management of the Maxillofacial Burn Patient: Current Therapy. Journal of Oral and Maxillofacial Surgery, 63, 247-252. https://doi.org/10.1016/j.joms.2004.03.019

[5] Haddock, N. and Levine, J. (2009) Breast Reconstruction with Implants, Tissue Expanders and AlloDerm: Predicting Volume and Maximizing the Skin Envelope in Skin Sparing Mastectomies. The Breast Journal, 16, 14-19. https://doi.org/10.1111/j.1524-4741.2009.00866.x

[6] Gryskiewicz, J.M., Rohrich, R.J. and Reagan, B.J. (2001) The Use of AlloDerm for the Correction of Nasal Contour Deformaties. Plastic and Reconstructive Surgery, 107, 561-570. https://doi.org/10.1097/00006534-200102000-00040

[7] Wax, M.K., Winslow, C.P. and Andersen, P.E. (2002) Use of Allogenic Dermis for Radial Forearm Free Flap Donor Site Coverage. Journal of Otolaryngology, 31, 341-345. https://doi.org/10.2310/7070.2002.34423 
[8] Albo, D., Awad, S.S., Berger, D.H. and Bellows, C.F. (2006) Decelularized Human Cadveric Dermis Provides a Safe Alternative for Primary Inguinal Hernia Repair in Contaminated Surgical Fields. The American Journal of Surgery, 192, e12-e17. https://doi.org/10.1016/j.amjsurg.2006.08.029

[9] Shorr, N., Perry, R.D., Goldberg, R.A., Hoenig, J. and Shorr, J. (2000) The Safety and Applications of Acellular Human Dermal Allograft in Ophthalmic Plastic and Reconstructive Surgery: A Preliminary Report. Ophthalmic Plastic \& Reconstructive Surgery, 16, 223-230. https://doi.org/10.1097/00002341-200005000-00010

[10] Harris, R.J. (2004) Gingival Augmentation with an Acellular Dermal Matrix: Human Evaluation of a Case-Placement of the Graft on Periosteum. The International Journal of Periodontics and Restorative Dentistry, 24, 378-385.

[11] Lee, J.M. and Boughner, D.R. (1985) Mechanical Properties of Human Pericardium. Differences in Viscoelastic Response When Compared with Canine Pericardium. Circulation Research, 57, 475-481. https://doi.org/10.1161/01.RES.57.3.475

[12] McGuire, D.A. and Hendricks, S.D. (2009) Allograft Tissue in ACL Reconstruction. Sports Medicine and Arthroscopy Review, 17, 224-233.

https://doi.org/10.1097/JSA.0b013e3181c0cf8a

[13] Ward, R.M., Sung, V.W., Clemons, J.L. and Myers, D.L. (2007) Paravaginal Repair with an AlloDerm Graft. American Journal of Obstetrics \& Gynecology, 197, c1-c5. https://doi.org/10.1016/j.ajog.2007.08.067

[14] Moore, M.A., Samsoll, B., Wallis, G., Triplett, S., Chen, S., Linhurst Jones, A. and Qin, X. (2015) Decellulariztion of Human Dermis Using Non-Denaturing Anionic Detergent and Endonuclease: A Review. Cell Tissue Bank, 16, 2489-259. https://doi.org/10.1007/s10561-014-9467-4

[15] Ewelda, A.M. and Marei, M.K. (2015) Naturally Occurring Extracellular Matrix Scaffolds for Dermal Regeneration: Do They Really Need Cells? Biomed Research International, 2015, Article ID: 839694.

[16] Deekan C.R. and Lake, S.P. (2017) Mechanical Properties of the Abdominal Wall and Biomaterials Utilized for Hernia Repair. Journal of the Mechanical Behavior of Biomedical Materials, 74, 411-417. https://doi.org/10.1016/j.jmbbm.2017.05.008

[17] Kato, Y.P., Dunn, M.G., Zawadsky, J.P., Tria, A.J. and Silver, F.H. (1991) Regeneration of Achilles Tend on Using a Collagen Tendon Prosthesis: Results of a Year Long Implantation Study. Journal Bone and Joint Surgery, 73-A, 561. https://doi.org/10.2106/00004623-199173040-00013

[18] Doillon, C.J., Silver, F.H., Olson, R.M., Kamath, C.Y. and Berg, R.A. (1988) Fibroblast and Epidermal Cell Type I Collagen Interactions: Cell Culture and Human Studies. Scanning Electron Microscopy, 2, 985-992.

[19] Terzini, M., Bignardi, C., Catagnoli, C., Cambieri, I., Zanetti, E.M. and Audenino A.L. (2016) Ex Vivo Dermis Mechanical Behavior in Relation to Decellularization Treatment Length. The Open Biomedical Journal, 10, 34-42. https://doi.org/10.2174/1874120701610010034

[20] Birk, D.E. and Silver, F.H. (1984) Collagen Fibrillogenesis In Vitro: Comparison of Types I, II and III. Archives Biochemistry and Biophysics, 235, 178-185. https://doi.org/10.1016/0003-9861(84)90266-2

[21] Silver, F.H., Freeman, J. and Seehra, G.P. (2003) Collagen Self-Assembly and Development of Matrix Mechanical Properties. Journal of Biomechanics, 36, 1529-1553. https://doi.org/10.1016/S0021-9290(03)00135-0

[22] Bender, E., Silver, F.H. and Hayashi, K. (1983) Model Conformations of the Car- 
boxylic Telopeptides In Vivo Based on Type I Collagen Fibril Banding Patterns. Collagen and Related Research, 3, 407-418. https://doi.org/10.1016/S0174-173X(83)80021-1

[23] Shah, R., DeVore, D., Pierce M.G. and Silver, F.H. (2016) Morphomechanics of Dermis-A Method for Non-Destructive Testing of Collagenous Tissues. Skin Research and Technology, 23, 399-406. https://doi.org/10.1111/srt.12349

[24] Shah, R., Pierce, M.C. and Silver, F.H. (2016) A Method for Non-Destructive Mechanical Testing of Tissues and Implants. Journal of Biomedical Materials Research Part $A, 105,5-22$.

[25] Shah, R.G., DeVore, D., Pierce, M.G. and Silver, F.H. (2017) Vibrational Analysis of Implants and Tissues: Calibration and Mechanical Spectroscopy of Multi-Component Materials. Journal of Biomedical Materials Research Part A, 105, 1666-1671. https://doi.org/10.1002/jbm.a.36041

[26] Silver, F.H. (2006) Mechanosensing and Mechanochemical Transduction in Extracellular Matrix. Springer, New York.

[27] Silver, F.H. and Quintero, L. (2003) Comparison of the Histologic Responses Observed at the Arterial Puncture Site after Employing Manual Compression and a New Collagen to Achieve Hemostasis. Cath Lab Digest, 11, 52-58.

[28] Dunn, M.G. and Silver, F.H. (1983) Viscoelastic Behavior of Human Connective Tissues: Relative Contribution of Viscous and Elastic Components. Connective Tissue Research, 12, 59-70. https://doi.org/10.3109/03008208309005612

[29] Seehra, G.P. and Silver, F.H. (2006) Viscoelastic Properties of Acid- and Alkaline-Treated Human Dermis: A Correlation between Total Surface Charge and Elastic Modulus. Skin Research and Technology, 12, 190-198. https://doi.org/10.1111/j.0909-752X.2006.00150.x

[30] Silver, F.H., Freeman, J. and DeVore, D. (2001) Viscoelastic Properties of Human Skin and Processed Dermis. Skin Research and Technology, 7, 18-23. https://doi.org/10.1034/j.1600-0846.2001.007001018.x

[31] Shah, R.G. and Silver, F.H. (2017) Viscoelastic Behavior of Tissues and Implant Materials: Estimation of the Elastic Modulus and Viscous Contribution Using Optical Coherence Tomography and Vibrational Analysis. Journal of Biomedical Technology and Research, 3, 105-109. 